\title{
Acute Visual Loss
}

\author{
Shirley H. Wray, MD, PhD, FRCP ${ }^{1}$ \\ ${ }^{1}$ Department of Neurology, Massachusetts General Hospital, Boston, \\ Massachusetts \\ Address for correspondence Shirley H. Wray, MD, PhD, FRCP, \\ Department of Neurology, Massachusetts General Hospital, 55 Fruit St, \\ Boston 02114, MA (e-mail: wray@helix.mgh.harvard.edu).
}

Semin Neurol 2016;36:425-432.
Abstract
Keywords
- ocular stroke
Acute visual loss is a frightening experience, a common ophthalmic emergency, and a diagnostic challenge. In this review, the author focusses on the diagnosis of transient monocular blindness and visual loss due to infarction of the retina and/or the optic nerve -the ocular parallel of cerebral stroke.

\section{Illustrative Case}

Day 1: The patient is a 75-year-old ophthalmologist who experienced an acute transient "white out" of her vision in her left eye lasting for 20 minutes. She had no accompanying symptoms. At this time, the patient was concerned and anxious that the white out of vision was an attack of transient monocular blindness-a transient ischemic attack that can herald stroke.

Day 4: She asked her ophthalmology fellow to examine her eye including intraocular pressure, dilated funduscopy, and automated (Humphrey) visual fields. The examination was completely normal apart from a suspicion that the left eye had a relative afferent pupil defect (RAPD) noted during the swinging flash light test, suggesting an optic neuropathy.

Day 8: She consulted a neuro-ophthalmologist who took a detailed history which revealed that at the time of the white out, the patient was reading a book laid open flat on a table. The entire left page suddenly went white such that the text was light gray and barely visible. The right-hand page however, retained black text. She did not cover either eye individually to determine if there was a visual loss in the homonymous nasal field of the right eye.

Her past history was negative for transient visual loss, migraine aura and/or headache, eye pain, speech disturbance, and/or symptoms affecting the extremities. Her medications were simvastatin (hyperlipidemia), alprazolam (anxiety), Naprosyn (disc pain), metoprolol (benign atrial arrhythmia), and occasional tramadol (pain from a ruptured disk).

The neuro-ophthalmological examination was normal and a diagnosis of a migraine visual event was made based on this history.

Day 11: Brain magnetic resonance imaging (MRI) without contrast was performed. The study showed a 2 -mm aneurysm in the left supraclinoid internal carotid artery (ICA) just proximal to the origin of the left posterior communicating artery.

Day 22: The patient consulted a neurovascular surgeon who obtained a head and neck computed tomographic angiogram that showed that the ICA aneurysm was unchanged in size and morphology from the previous exam. No other aneurysm was seen. The surgeon reviewed all the imaging studies with the patient and reassured her that there was minimal risk of rupture of the aneurysm.

\section{Special Explanatory Note}

The swinging flashlight test is probably the most valuable clinical test of optic nerve dysfunction available for all physicians. The test is used to test the integrity of the afferent light pathway by comparing the constriction to direct light of one pupil versus the other. The flashlight should be moved rhythmically across the bridge of the nose to direct the light onto each eye for 1 to 2 seconds. With a RAPD of the left eye, both the pupils constrict when the light is shone on the right eye. When the light is swung back to the left eye, both pupils dilate, resulting in the suspicion that the left optic nerve is not functioning properly and there is a paradoxical dilation of the left pupil.

The migrainous nature of transient neurologic manifestations (accompaniments), such as diplopia, monocular blindness, and hemianopia, is accepted when they occur repeatedly in association with headache in patients under the age of 30 . It is a different matter when similar phenomena occur for the first time after the age of 40 when differentiation from the transient ischemic attacks (TIAs) of cerebrovascular disease may be necessary; a good example is the case history presented here. ${ }^{1}$

\section{Acute Transient Monocular Blindness}

Transient loss of vision in one eye, termed amaurosis fugax or transient monocular blindness (TMB), is the single most
Issue Theme Pearls and Pitfalls, Part 2; Guest Editors, Justin C. McArthur, MBBS, $\mathrm{MPH}, \mathrm{FAAN}, \mathrm{FANA}$, and Nicoline Schiess, $\mathrm{MD}, \mathrm{MPH}$
Copyright (c) 2016 by Thieme Medical Publishers, Inc., 333 Seventh Avenue, New York, NY 10001, USA. Tel: +1(212) 584-4662.
Dol http://dx.doi.org/ $10.1055 / \mathrm{s}-0036-1585452$. ISSN 0271-8235. 
important visual symptom of arteriosclerotic vascular disease, arteritis and states of altered coagulability, and thrombocytosis.

A detailed description of the attack and duration of the visual disturbance permits classification of the TMB event into one of four types. Type I is due to transient retinal ischemia, type II to retinal vascular insufficiency, type III to vasospasm, and type IV occurs in association with antiphospholipid antibodies as well as cases of unknown etiology (-Table 1). ${ }^{2}$

\section{Transient Monocular Blindness Type I}

Type I is characterized by a sudden brief attack of partial or complete dimming or loss of vision, lasting seconds to minutes, with total recovery. Partial impairment is described as a grayout, or as an ascending or descending curtain or blind moving sideways across the visual field. Occasionally, the patient will describe moving tracks of light. Ipsilateral headache is rare. In 1952, Fisher drew attention to the association of TMB of this brevity with contralateral hemiplegia. ${ }^{3}$

Transient monocular blindness is regarded as one variety of carotid artery TIA, and like other TIAs, TMB should be recognized as a warning of an impending stroke. Physical examination of the blood vessels of the head and neck and eyes is key to the detection of signs localizing the lesion to the internal carotid artery (ICA).

\section{Clinical Signs}

- Visual acuity: Normal corrected vision

- Visual field: Full

- Pupil: Normal reflexes

- Funduscopy (pupils dilated): A normal funduscopic examination is the most common finding. Retinal emboli are diagnostic.

- Retinal emboli differ in appearance depending on their constituents. Fisher, in a detailed classical description, followed emboli funduscopically for many hours and watched them traverse the retinal arteries. ${ }^{4}$
- Cholesterol emboli (Hollenhorst plaques), are bright, yellowish glinting lipid emboli (-Fig. 1). Hollenhorst plaques are virtually diagnostic of localized ICA occlusive disease. ${ }^{5,6}$ Typically, an ipsilateral carotid bruit is present, aortic or cardiac disease are absent, and there are no exogenous sources of emboli, for example, intravenous drug use, severe trauma, or the injection of cosmetic facial fillers (such as autologous fat, collagen, or hyaluronic acid) ${ }^{7}$

A cholesterol embolus is a poor prognostic sign: $93 \%$ of such patients have vascular disease at presentation, $15 \%$ die within the first year, and 55\% in 7 years. The cause of death is heart disease, $6: 1$ over stroke. $^{8}$

- Calcific emboli are characteristically matte-white, nonscintillating, and somewhat wider than the blood column. Calcific emboli may be dislodged by surgical manipulation of calcified heart valves.

- Platelet emboli are pale nonscintillating white emboli and tend to be multiple.

- Fibrin plugs resemble platelet emboli, and may originate from mural plaques following myocardial infarction.

- Asymmetric hypertensive retinopathy: In a hypertensive patient, funduscopy may show asymmetric hypertensive retinopathy. The caliber of the retinal arteries on the side of an ICA stenosis may be reduced and will show fewer hypertensive changes than the retinal vessels of the contralateral eye. Focal cotton wool spots (cystoid bodies), in the absence of hypertensive retinopathy, are due to embolic microinfarction, and may be seen when no emboli are visible (-Table $\mathbf{2}$ ).

- Bruit: The typical carotid bruit is a high-pitched, long, very focal sound heard loudest over the carotid bifurcation. When a bruit at the bifurcation is also audible over the ipsilateral eye, the examiner can be confident that the bruit is of ICA origin and that the artery is patent. An ipsilateral ocular bruit alone may indicate stenosis of the intracavernous segment of the ICA. When flow in the ICA is

Table 1 Types of transient monocular blindness

\begin{tabular}{|l|l|l|l|l|}
\hline & Type I & Type II & Type III & Type IV \\
\hline Onset & Abrupt & Less rapid & Abrupt & Abrupt \\
\hline Visual field & All or partial & All or partial & $\begin{array}{l}\text { All or progressive } \\
\text { contraction }\end{array}$ & Resembles types I, II, or III \\
\hline Visual loss & $\begin{array}{l}\text { May black } \\
\text { out completely }\end{array}$ & $\begin{array}{l}\text { Loss of contrast vision, photopsia, } \\
\text { sunlight provoked }\end{array}$ & $\begin{array}{l}\text { May spare fixation, } \\
\text { photopsia, } \\
\text { scintillating sparkles }\end{array}$ & May alternate between eyes \\
\hline Length & Seconds or minutes & Minutes or hours & Minutes & Any length \\
\hline Recovery & Complete & Complete & Usually complete & Complete \\
\hline Pain & No & Rare & Often & No \\
\hline Mechanism & $\begin{array}{l}\text { Retinal ischemia, } \\
\text { embolus, arteritis }\end{array}$ & $\begin{array}{l}\text { Retinal hypoperfusion, } \\
\text { carotid occlusive disease }\end{array}$ & Vasospasm migraine & APS Idiopathic \\
\hline
\end{tabular}




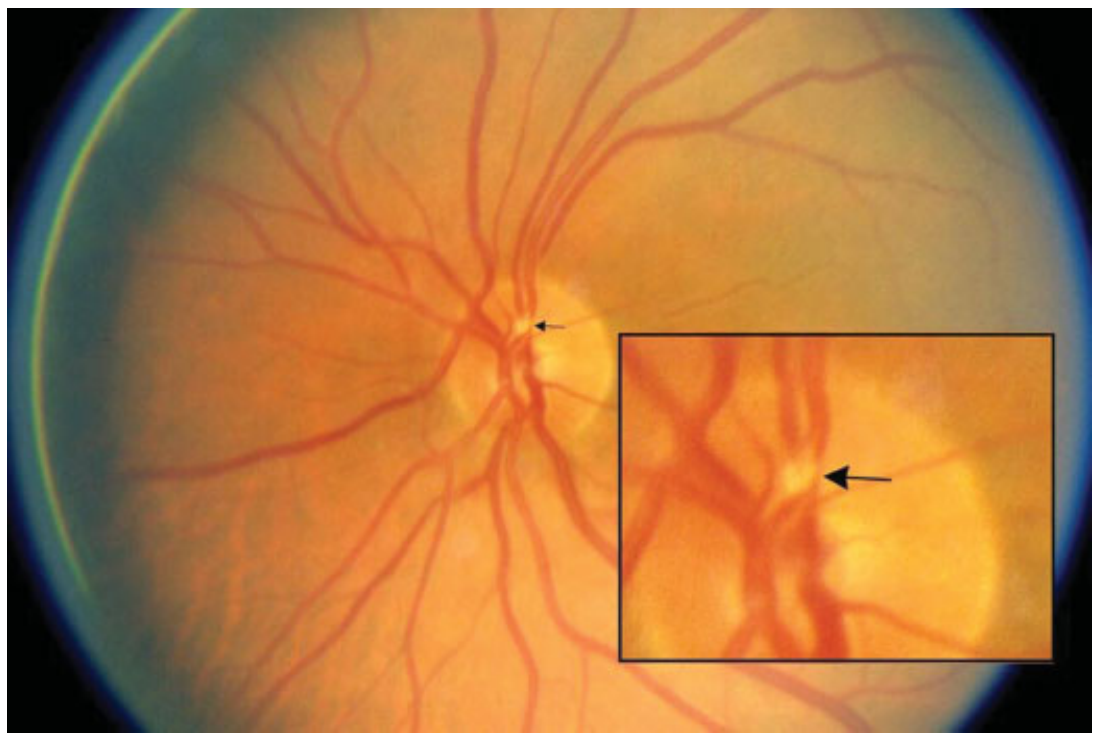

Fig. 1 Hollenhorst plaque, bright yellow glinting cholesterol embolus, impacted in an artery on the surface of the disc (arrow).

severely diminished, no bruit may be audible. A bruit can also arise from the proximal external carotid artery (ECA), in which case it usually radiates toward the jaw and can be diminished by pressure on ECA branches.

- Anisocoria: Anisocoria, with normal pupil reflexes, is an important sign of ICA dissection. Miosis and partial ptosis in the ipsilateral eye indicates an oculosympathetic palsy, referred to as Horner's syndrome. Horner's syndrome results from damage to the autonomic nerve fibers in the ICA sheath in the neck. Facial sweating is preserved because sympathetic innervation of sweat glands travels along the ECA. The presenting symptom of ICA dissection is pain. Ipsilateral neck pain, headache, and pain in the face or retro-orbital pain may occur.

\section{Transient Monocular Blindness Type II}

Type II occurs in extensive extracranial occlusive disease involving both the ipsilateral ICA and ECA due to retinal vascular insufficiency. The visual disturbance is different from type I TMB. Typically, the attack is less rapid in onset (over 5 minutes), longer in duration (minutes to hours), and recovery is gradual (-Table $\mathbf{1}$ ). Visual acuity is not altered significantly during the episode, but contrast acuity is. Bright

Table 2 Funduscopic signs in transient monocular blindness

\begin{tabular}{|l|}
\hline Normal disc and fundus \\
\hline Retinal emboli \\
\hline Branch retinal artery occlusion \pm visible embolus \\
\hline Retinal infarct (cytoid body) \pm hemorrhage \\
\hline Venous stasis retinopathy \\
\hline Asymmetric hypertensive retinopathy \\
\hline Ischemic disc swelling \\
\hline transient monocular blindness \\
\hline
\end{tabular}

objects appear brighter, dark objects become more difficult to see, and the edges of bright objects can appear to flicker. When the bright dazzling sensation is marked, the overall effect is one of overexposure and the patient may experience difficulty reading because of the dazzle of white paper. When sight becomes fragmented and patchy, patients describe the appearance as a photographic negative.

The conditions that provoke attacks of type II TMB are systemic hypotension, venous hypertension, and extracerebral steal. The attacks occur on stooping or straining when venous pressure rises, on standing, or during exercise, and on exposure to bright lights or warm surroundings. ${ }^{9}$

\section{Clinical Signs}

- Visual acuity: Normal corrected vision

- Visual field: Full

- Pupil: Normal reflexes

- Funduscopy: Venous stasis retinopathy (pupils dilated). Type II's unusual visual symptoms are retinal in origin, and a low retinal arterial pressure is always present. Compensatory retinal venous changes occur and venous stasis retinopathy develops, indicating that reduction in flow in the ophthalmic artery is severe and long standing. The early signs of venous stasis retinopathy due to occlusive disease of the ICA and ECA are microaneurysms, multiple small blot-like intraretinal hemorrhages in the midperiphery, segmental narrowing and dilation of the veins, spontaneous pulsations of retinal arteries on the disc, and ischemic disc swelling. ${ }^{10}$ The fundus appearance resembles diabetic retinopathy, but is easily distinguished from diabetes by being unilateral. Signs of anterior segment ischemia may also be present.

Transient Monocular Blindness Type III

Type III is rare. It is due to vasospasm. ${ }^{11}$ Temporary vasospasm offers a plausible explanation of TMB in migraineurs. However, monocular migraine vasospasm can only be 
diagnosed when constriction of the retinal arteries is visualized funduscopically during a TMB attack. It may be suspected when all other causes of TMB are excluded and examination of the eye is normal.

\section{Transient Monocular Blindness Type IV}

Type IV cases include young people with episodic TMB resembling the visual obscurations of type I, except in the duration of the attack which frequently is too long (30-60 minutes) or too short (seconds only). Occasionally, the visual disturbance resembles the temporary loss of contrast vision typical of type II or the photopsias and scintillations of vasospastic type III. In several studies and in our own experience, no cause has been found to explain recurrent TMB type IV in young adults, apart from observations by Digre et $\mathrm{al}^{12}{ }^{12}$ which document the association of TMB type IV in patients with anticardiolipin antibodies.

\section{Acute Persistent Monocular Blindness}

Acute persistent monocular blindness is the major symptom of an ocular stroke. Ocular stroke may be due to occlusion of the terminal branches of the ophthalmic artery, the central retinal artery, or the posterior ciliary arteries which leads to infarction of the retina and/or the optic nerve. Three common ocular strokes are central retinal artery occlusion, branch retinal artery occlusion, and anterior ischemic optic neuropathy.

\section{Central Retinal Artery Occlusion}

Central retinal artery occlusion (CRAO) can occur at any age and is characterized by apoplectic loss of vision. Eye pain is atypical, but when present, it suggests involvement of the ophthalmic artery. Transient monocular blindness may precede the occlusion, and attacks of TMB may increase in frequency over 12 to 24 hours before blindness occurs: crescendo TMB.

Approximately 15 to $30 \%$ of the general population has a cilioretinal artery, a branch of the short posterior ciliary artery. It supplies a part or the whole of the fovea, and in those eyes where there is a CRAO, the cilioretinal artery is spared and visual acuity preserved at $20 / 50$ or better (-Fig. 2).

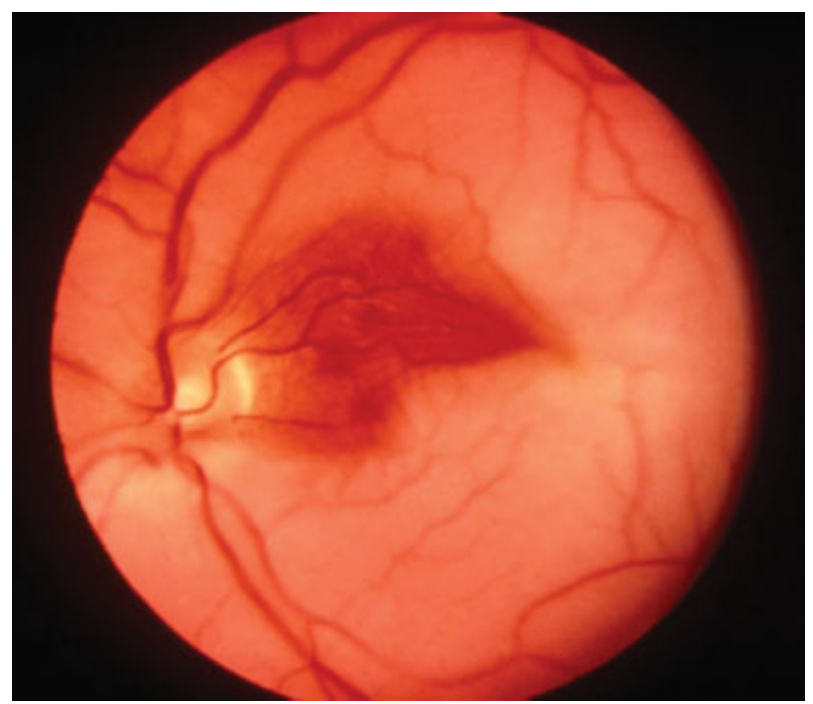

Fig. 2 Central retinal artery occlusion with cilioretinal artery sparing.
The principle causes of CRAO are

1. Embolic obstruction

2. Occlusion in situ, due to thrombosis or hemorrhage into an atheromatous plaque

3. Inflammatory arteritis-giant cell temporal arteritis (TA), thromboangiitis obliterans, and polyarteritis nodosa with involvement of the choroidal and retinal arteries

4. Angiospasm (e.g., Raynaud's disease) or migraine

5. Arterial occlusion that occurs hydrostatically with either

a. The high intraocular pressure of glaucoma

b. The low retinal blood pressure of carotid stenosis or the aortic arch syndrome

c. Severe hypotension

Optical coherence tomography can be helpful in showing the extent of infarction of the inner retinal layers in patients with CRAO). ${ }^{13}$

\section{Clinical Signs}

- Visual acuity: Is variable-no light perception or response to light \pm hand movement.

- Visual field: To light present diffusely

- Pupil: An amaurotic pupil, an absence of constriction to light on direct illumination, intact consensual light response, and intact near response, is said to be present when the eye is completely blind. An afferent pupil defect, an impaired direct light response, is present if some vision is preserved, even hand motion vision.

- Funduscopy (pupils dilated): Retinal signs are dependent on the time that the fundus is examined. Initial signs are few: boxcar segmentation with slow "streaming" of blood flow in the retinal veins, a normal optic disc, and no hemorrhages or exudates (-Fig. 3A). Total obstruction posterior to the laminae cribrosa should be suspected when the retinal arteries on the disc start to pulsate with gentle pressure on the globe, indicating a very low retinal diastolic pressure. After an hour or more, the characteristic diagnostic fundus changes are seen. The ischemic retina takes on a white ground glass appearance, and the normal red color of the choroid showing through at the fovea is accentuated and visualized as a central cherry-red spot at the macula (-Fig. 3B). Within days of the acute event, the retinal opacification, the cherry-red spot, and the nerve fiber layer striations disappear and optic atrophy of the optic disc of the primary type develops.

- Intraocular pressure: When the signs of CRAO are secondary to an ophthalmic artery occlusion, the intraocular pressure in the eye is low and the cherry red spot at the macula is less vivid.

- Retinal emboli: Transient monocular blindness as a premonitory symptom of CRAO suggests an embolic cause or temporal arteritis. For patients under the age of 40 years, the sources of retinal emboli include cardiac disease, in particular rheumatic valvular disease, bacterial endocarditis, cardiac myxoma, ${ }^{14}$ and ipsilateral ICA dissection. ${ }^{15}$ Over age 40 years, the sources of emboli include cardiac disease, atheroma of the aorta and diffuse disseminated 

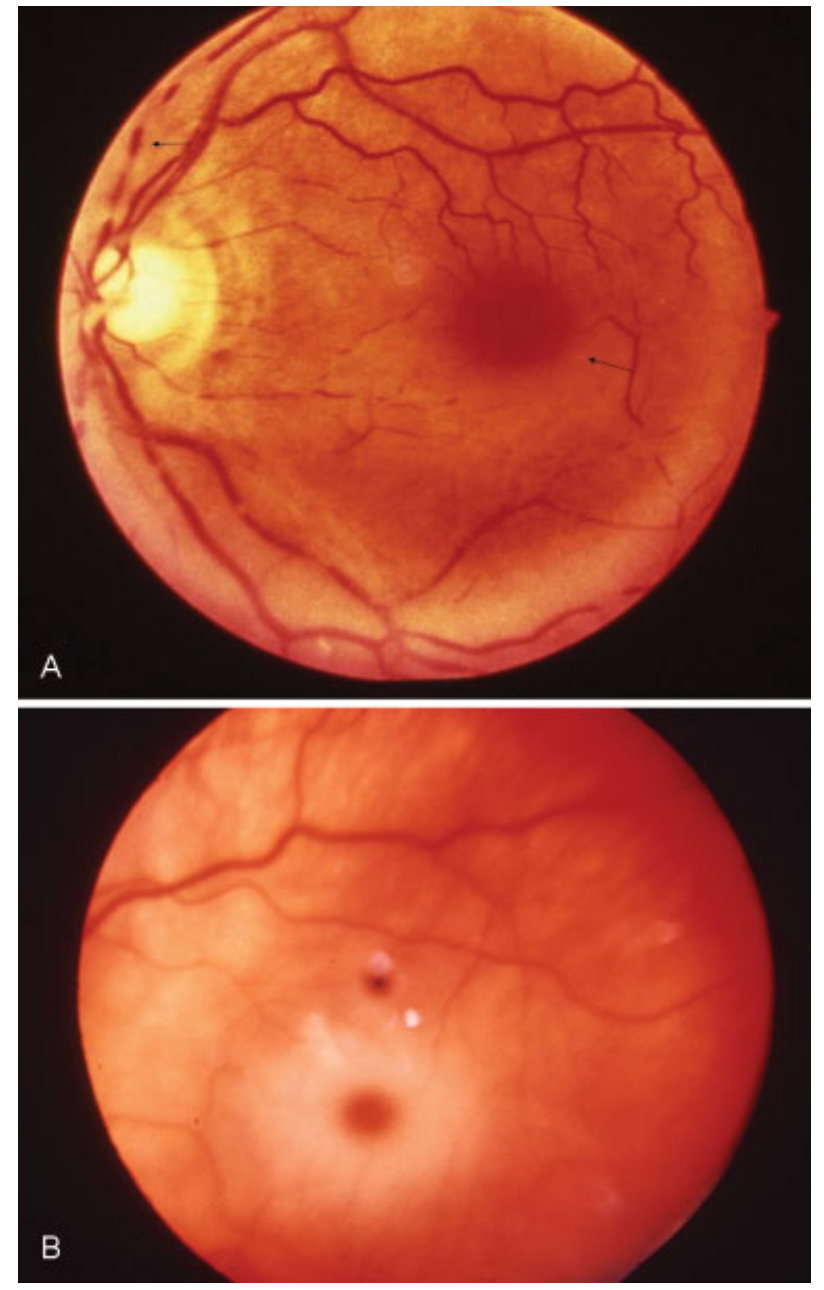

Fig. 3 Central retinal artery occlusion. (A) Acute boxcar segmentation (arrow) and slow streaming of blood in the veins. (B) Macula cherry red spot enhanced by infarction of the retinal ganglion cell layer.

atheroembolism, ipsilateral ICA atheromatous disease, and ICA dissection. ${ }^{15}$

- Cardiovascular signs: The most important additional signs to look for are absence of a temporal artery pulse and/or tenderness over the vessel on palpation. A CRAO may be the only symptom of temporal arteritis in 5 to $10 \%$ of elderly patients, and the risk of blindness in the fellow eye is extremely high.

Fundus fluorescence angiography (FFA) and inflammatory markers, such as erythrocyte sedimentation rate, C-reactive protein (CRP), and platelet count, should be performed. In GCA, ESR is usually $>50$, and may $>100$, but in 5 to $10 \%$ of cases will be normal. Elevated CRP has higher sensitivity and specificity ${ }^{16}$ ). Arteritic CRAO cases should be treated promptly with intravenous high-dose steroids to prevent blindness of the contralateral eye. ${ }^{17}$

\section{Branch Retinal Artery Occlusion}

A branch retinal artery occlusion (BRAO) is characterized by sudden persistent loss of vision in a sector of the visual field with infarction of an area of the retina corresponding to the vascular territory supplied by the occluded artery.
Susac's syndrome, a rare microangiopathy primarily affecting young women between the ages of 21 and $41,{ }^{18}$ is an important cause of branch artery occlusions. This syndrome, reported by Susac et al in $1979,{ }^{19}$ consists of the triad encephalopathy, BRAOs, and neurosensory hearing loss.

Branch artery occlusions are present in $40 \%$ of cases and may be quite extensive and spectacular or subtle. They tend to be bilateral and multiple, causing scotoma or photopsias, and are recurrent.

The majority of patients do not present with the complete triad. An appropriate diagnostic approach is to search for absent components of the triad by getting brain MR diffusion weighted imaging, which almost always shows involvement of the corpus callosum in the encephalopathic patient, and audiometry to detect deafness. ${ }^{20}$ These tests together with fundus fluorescein angiography are considered crucial tests to aid in the diagnosis of Susac's syndrome.

The pathogenesis of Susac's microangiopathy is unknown. Currently, the syndrome is thought to be an immunomediated endotheliopathy that affects the microvasculature of the retina, brain, and inner ear. A small proportion of patients have been found to have antiendothelial cell antibodies.

\section{Clinical Signs}

- Visual acuity: Normal corrected vision

- Visual field: Arcuate nerve fiber defect or sector loss consistent with focal retinal infarction

- Pupil: Normal reflexes

- Funduscopy (pupils dilated): Typically shows an embolus impacted at the bifurcation of a branch artery and pallor of the infarcted retina distal to the location of the embolus (-Fig. 4A).

- If no embolus is found, funduscopy in BRAO due to retinal microangiography might show yellow-white retinal wall plaques (Gass bodies ${ }^{21,22}$ located at midarterial segments proximal to sites of occlusion and distant from retinal arterial bifurcations ( - Fig. 4B). Gass bodies reflect damage to the endothelium and should not be mistaken for emboli (-Fig. 4C).

- Hyperfluorescence of the arterial wall and leakage of dye consistently detected by fluorescein angiography is a biomarker of disease activity, and present even when the patient is asymptomatic. ${ }^{23}$

\section{Anterior Ischemic Optic Neuropathy}

Ischemic optic neuropathy may be arteritic or nonarteritic, anterior and posterior.

Nonarteritic anterior ischemic neuropathy (NA-AION), the most common form, ${ }^{24-27}$ is characterized by acute, monocular, painless visual loss, and swelling of the optic disc ( - Fig. 5A). The process leading to NA-AION appears to be complex and multifactorial. ${ }^{28}$ The precise cause is unknown. A small cup-to-disc ratio ("disc at risk") may be a risk factor. ${ }^{29}$

Nonarteritic AION in elderly patients is commonly accompanied by cerebral stroke risk factors: hypertension, hyperlipidemia, diabetes mellitus, obesity, and smoking. Nonarteritic AION may occur in collagen vascular diseases such as systemic lupus erythematosus and polyarteritis nodosa, or as a complication of 

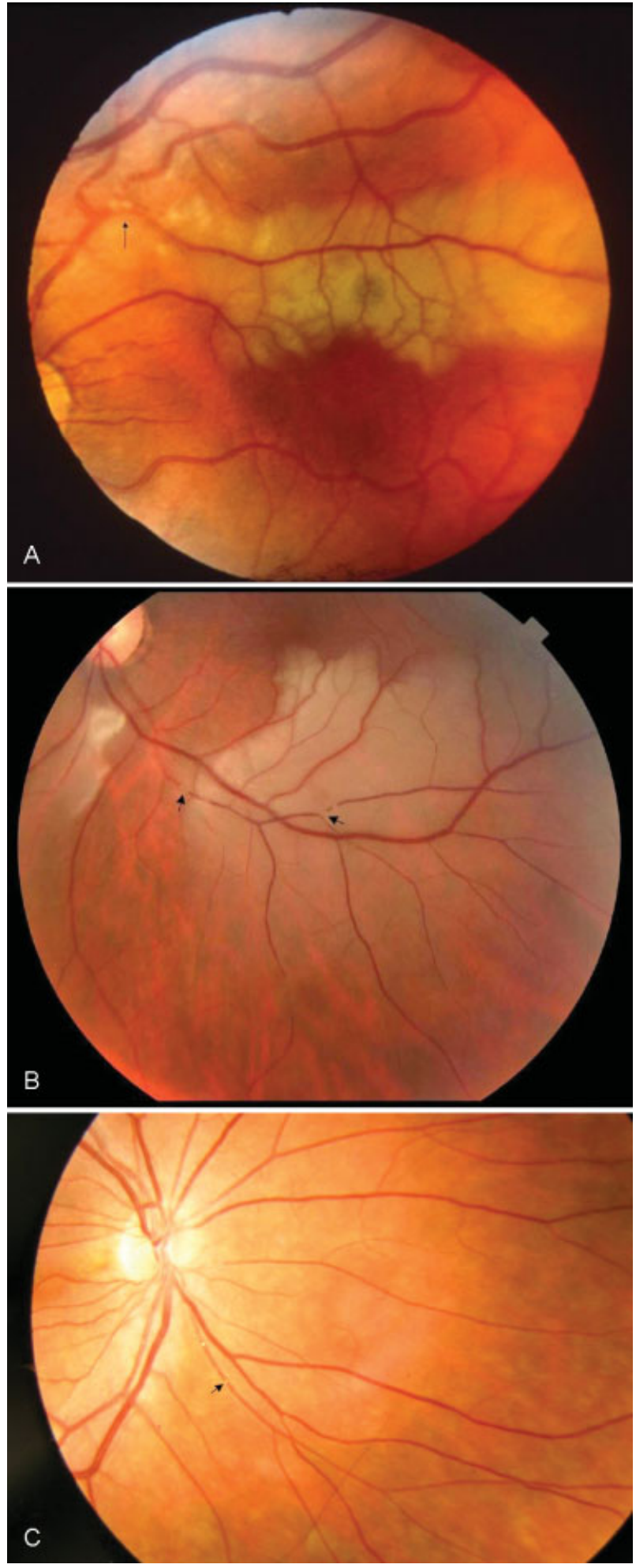

Fig. 4 Branch retinal artery occlusion (BRAO). (A) Embolic BRAO, two emboli (arrow) impacted at the bifurcation of an artery with infarction of the retina distal to the site of obstruction. (B) Susac BRAO due to retinal microangiopathy with yellow-white retinal wall plaques (GASS bodies) in midarterial segments distal from retinal arterial bifurcations (arrows). (Courtesy of Dr. Ivana Vodopivec)

(C) Gass bodies, small bead-like bodies in the arterial wall (arrow) indicative of endothelial damage, which should not be mistaken for emboli. (Courtesy of Dr. Ivana Vodopivec)
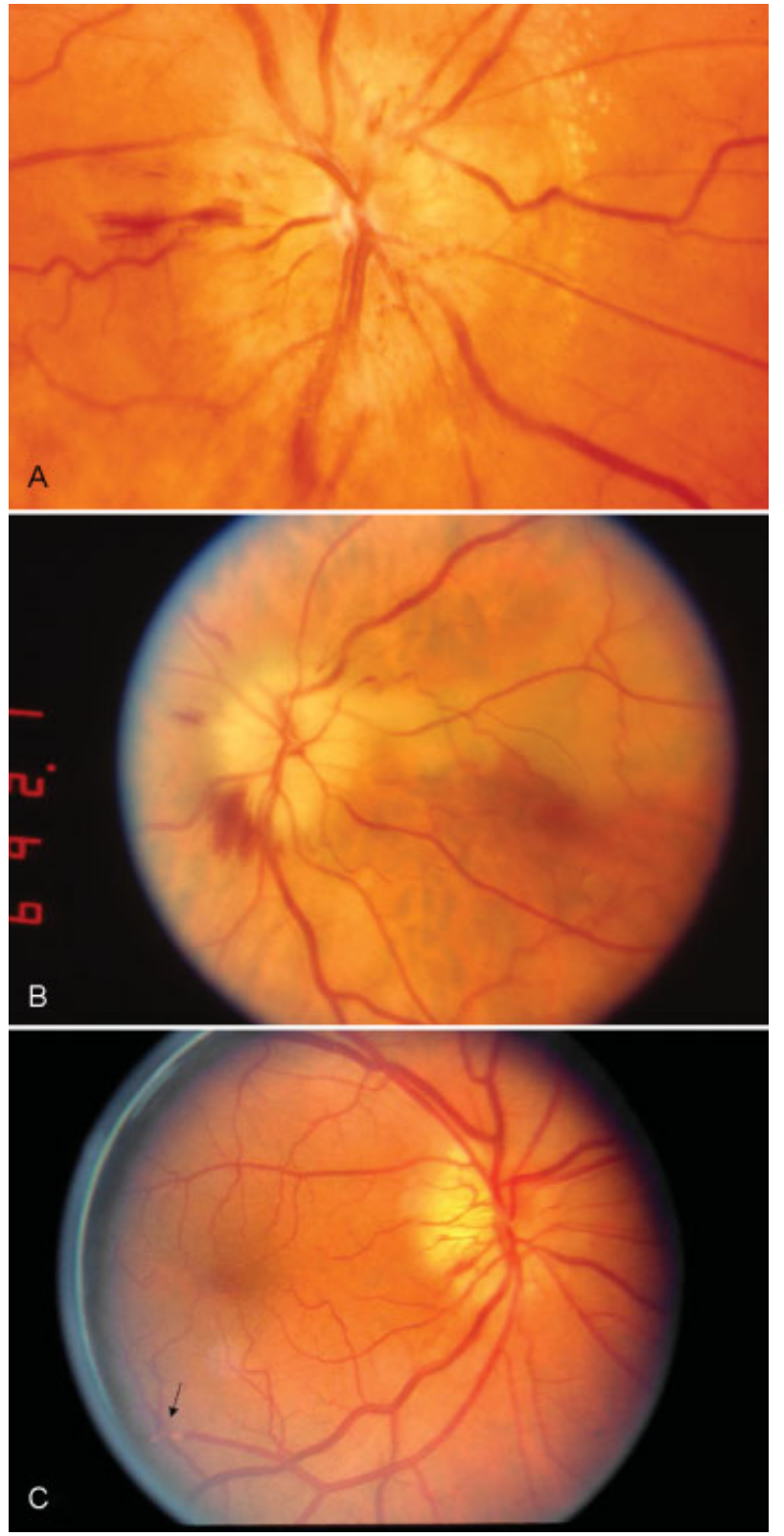

Fig. 5 Anterior ischemic neuropathy (AION). (A) Nonarteritic AION with acute ischemic swelling of the disc and multiple flame-shaped hemorrhages. (B) Arteritic AION with acute ischemic swelling, pallor of the disc, and blot hemorrhage. (C) Embolic AION with ischemic disc swelling, peripapillary flame-shaped hemorrhage, and a retinal embolus.

arterial hypotension during surgical procedures, renal hemodialysis, ${ }^{30}$ or massive hemorrhage. Other etiologies include hematological disorders such as sickle-cell trait, polycythemia, thrombocytopenic purpura, leukemia, and various types of anemia. Recent observations suggest that obstructive sleep apnea may play a role owing to the relative ischemia that occurs during apneic episodes. ${ }^{31}$ Sequential NA-AION is reported in patients taking sildenafil and related drugs for erectile dysfunction. ${ }^{32}$ About $50 \%$ of patients lose vision in the fellow eye after an interval of months to years. 
A diagnostic challenge is to differentiate arteritic AION due to temporal arteritis (TA-AION) from NA-AION to prevent progressive blindness. Although the clinical presentation of TA-AION mimics the symptoms and signs of NA-AION, numerous red flags such as headache, jaw claudication, neck pain, and scalp tenderness should raise the suspicion that the patient has active arteritis. However, approximately $25 \%$ of patients with TA-AION present as isolated ischemic optic neuropathy with no systemic arteritic complaints.

Embolic AION is rare, and visualization of a retinal embolus is diagnostic. We have seen three cases of AION combined with retinal and posterior ciliary artery emboli following coronary artery bypass surgery. Choroidal infarction was detected in each case by fluorescein angiography.

\section{Clinical Signs}

- Visual acuity: At presentation, two-fifths of affected eyes have an acuity of 20/60 or worse.

- Visual field: Sixty percent of visual field defects are altitudinal, with the inferior field being affected 3 times more frequently than the superior field. The reason for the preferential loss of the inferior field is unknown.

- Pupil: The affected eye will have an afferent pupil defect.

- Funduscopy (pupils dilated): In TA-AION, the disc typically appears acutely pale as well as swollen (-Fig. 5B). The ischemic swelling may be sectorial, involving the superior half of the disc in cases with an inferior altitudinal defect. Peripapillary blot or flame-shaped hemorrhages are characteristic.

The unique funduscopic sign of AION plus retinal embolus is shown in - Fig. $\mathbf{5 C}$.

- Temporal artery pulses: Tenderness on palpation of the ipsilateral temporal artery is diagnostic of temporal arteritis. Absent pulsation of the temporal artery pulse on one or both sides without tenderness over the artery should be considered due to temporal arteritis until proven otherwise.

\section{Conclusion}

The following are the clinical pearls to remember:

- Take a meticulous history.

- Think migraine when vision is obscured in both eyes by a positive illusion (e.g., white out, scintillating scotoma, photopsia, or colored clouds) that persist with eyes closed.

- The duration of visual loss classifies TMB into types I, II, III, and IV.

- Type I is due to retinal ischemia.

- Type II is due to retinal vascular insufficiency.

- Type III is due to vasospasm and results in loss of contrast vision.

- Type IV is associated with APS.

- Dilated funduscopy is mandatory.

- Hollenhorst plaques are diagnostic of ipsilateral ICA atheroma.
- Crescendo TMB may herald a CRAO.

- An FFA is mandatory in BRAOs

- Always check inflammatory markers in CRAO and AION.

- Register a patient with severe visual loss as legally blind so that they may qualify for assistance.

\section{References}

1 Fisher CM. Late-life migraine accompaniments as a cause of unexplained transient ischemic attacks. Can J Neurol Sci 1980; $7(1): 9-17$

2 Wray SH. Extracranial internal carotid artery disease. In: Bernstein EF, ed. Amaurosis fugax. New York, NY: Springer-Verlag; 1988:72-800

3 Fisher M. Transient monocular blindness associated with hemiplegia. AMA Arch Opthalmol 1952;47(2):167-203

4 Fisher CM. Observations of the fundus oculi in transient monocular blindness. Neurology 1959;9(5):333-347

5 Hollenhorst RW. The ocular manifestations of internal carotid arterial thrombosis. Med Clin North Am 1960;44:897-908

6 Hollenhorst RW. Significance of bright plaques in the retinal arterioles. JAMA 1961;178(1):23-29

7 Carle MV, Roe R, Novack R, Boyer DS. Cosmetic facial fillers and severe vision loss. JAMA Ophthalmol 2014;132(5): 637-639

8 Savino PJ, Glaser JS, Cassady J. Retinal stroke. Is the patient at risk? Arch Ophthalmol 1977;95(7):1185-1189

9 Furlan AJ, Whisnant JP, Kearns TP. Unilateral visual loss in bright light. An unusual symptom of carotid artery occlusive disease. Arch Neurol 1979;36(11):675-676

10 Kearns TP, Hollenhorst RW. Venous-stasis retinopathy of occlusive disease of the carotid artery. Proc Staff Meet Mayo Clin 1963; 38:304-312

11 Burger SK, Saul RF, Selhorst JB, Thurston SE. Transient monocular blindness caused by vasospasm. N Engl J Med 1991;325(12): 870-873

12 Digre KB, Durcan FJ, Branch DW, Jacobson DM, Varner MW, Baringer JR. Amaurosis fugax associated with antiphospholipid antibodies. Ann Neurol 1989;25(3):228-232

13 Falkenberry SM, Ip MS, Blodi BA, Gunther JB. Optical coherence tomography findings in central retinal artery occlusion. Ophthalmic Surg Lasers Imaging 2006;37(6):502-505

14 Cogan DG, Wray SH. Vascular occlusions in the eye from cardiac myxomas. Am J Ophthalmol 1975;80(3 Pt 1):396-403

15 Appen RE, Wray SH, Cogan DG. Central retinal artery occlusion. Am J Ophthalmol 1975;79(3):374-381

16 Hayreh SS, Podhajsky PA, Raman R, Zimmerman B. Giant cell arteritis: validity and reliability of various diagnostic criteria. Am J Ophthalmol 1997;123(3):285-296

17 Varma DD, Cugati S, Lee AW, Chen CS. A review of central retinal artery occlusion: clinical presentation and management. Eye (Lond) 2013;27(6):688-697

18 Susac JO. Susac's syndrome: the triad of microangiopathy of the brain and retina with hearing loss in young women. Neurology 1994;44(4):591-593

19 Susac JO, Hardman JM, Selhorst JB. Microangiopathy of the brain and retina. Neurology 1979;29(3):313-316

20 Vodopivec I, Venna N, Rizzo JF III, Prasad S. Clinical features, diagnostic findings, and treatment of Susac syndrome: a case series. J Neurol Sci 2015;357(1-2):50-57

21 Gass JDM, Tiedeman J, Thomas MA. Idiopathic recurrent branch retinal arterial occlusion. Ophthalmology 1986;93(9): 1148-1157

22 Egan RA, Hills WL, Susac JO. Gass plaques and fluorescein leakage in Susac syndrome. J Neurol Sci 2010;299(1-2):97-100

23 Egan RA, Ha Nguyen T, Gass JDM, Rizzo JF III, Tivnan J, Susac JO. Retinal arterial wall plaques in Susac syndrome. Am J Ophthalmol 2003;135(4):483-486 
24 Hayreh SS. Ischemic optic neuropathy. Prog Retin Eye Res 2009; 28(1):34-62

25 Hayreh SS. Ischemic optic neuropathies - where are we now? Graefes Arch Clin Exp Ophthalmol 2013;251(8):1873-1884

26 Banik R. Nonarteritic anterior ischemic optic neuropathy: an update on demographics, clinical presentation, pathophysiology, animal models, prognosis, and treatment. J Clin Exp Ophthalmol 2013;S3:004

27 Luneau K, Newman NJ, Biousse V. Ischemic optic neuropathies. Neurologist 2008;14(6):341-354

28 Hayreh SS. Ocular vascular occlusive disorders: natural history of visual outcome. Prog Retin Eye Res 2014;41:1-25
29 Hayreh SS, Zimmerman MB. Nonarteritic anterior ischemic optic neuropathy: refractive error and its relationship to cup/disc ratio. Ophthalmology 2008;115(12):2275-2281

30 Sabt BI. Anterior ischemic optic neuropathy and dialysis: effect of hypotension. Oman J Ophthalmol 2013;6(1):64-65

31 Archer EL, Pepin S. Obstructive sleep apnea and nonarteritic anterior ischemic optic neuropathy: evidence for an association. J Clin Sleep Med 2013;9(6):613-618

32 Galvez-Ruiz A, Arishi N. Sequential, non-arteritic anterior ischemic optic neuropathy in patients taking sildenafil: a report of ten cases. Saudi J Ophthalmol 2013;27(4):241-246 